Historic, Archive Document

Do not assume content reflects current scientific knowledge, policies, or practices. 



\author{
P. O. BOX 131 \\ Jamaica Plain, Boston, Mass.
}

\section{Special Quotation for Forestry Seed}

CONIFER SEED

\begin{abstract}
Abies balsamea, Balsam Fir....................... $\$ 2.651 \mathrm{~b}$.
Abies concolor, White Fir....................... $1.50 \ldots \ldots$ b.

Ginkgo biloba, fresh domestic seed .................... 1.25 lb.

Larix leptolepis, Japanese Larch ... . . . . . . . . . . . . . . . . 3.25 lb.

Picea canadensis, White Spruce, $80-90 \% \ldots \ldots \ldots \ldots \ldots \ldots \ldots .2 .25$ lb.

Picea, excelsa, Norway Spruce, $80-90 \%$, very best seed from North

Central Europe.......................... 1.50 lb.

Picea pungens, Colorado Spruce.................. $4.001 \mathrm{lb}$.

Pinus montana mughus, Mountain Pine................. $2.25 \mathrm{lb}$.

Pinus sylvestris, Scotch Pine, 80-90\%, straight trunk type from controlled forests in Eastern Germany, the heart of the Scotch

Pine Belt in Northern Europe.................. 4.00 lb.

Pinus strobus, American White Pine.................. $2.50 \mathrm{lb}$.

Pseudotsuga Douglasi glauca, hardy Colorado Douglas Fir....... 5.00 lb.

Thuja occidentalis, American Arborvitae, North Eastern Seed...... 3.25 lb.

Tsuga canadensis, Canadian Hemlock, New England Seed.......... 8.75 lb.
\end{abstract}

$\$ 210.00$ per $100 \mathrm{lbs}$.

125.00 per $100 \mathrm{lbs}$.

350.00 per $100 \mathrm{lbs}$.

210.00 per $100 \mathrm{lbs}$.

375.00 per $100 \mathrm{lbs}$. 225.00 per $100 \mathrm{lbs}$. 475.00 per $100 \mathrm{lbs}$. 300.00 per $100 \mathrm{lbs}$.

\section{HARDWOOD SEED}

Acer saccharum, Sugar Maple.

.$\$ 1.001 \mathrm{lb}$.

Gleditsia tricanthos, Honeylocust................... $85 \mathrm{lb}$.

Quercus coccinea, Scarlet Oak, Northern Seed, New England. 2.50 per $10 \mathrm{lbs}$. Quercus palustris, Pin Oak, Northern Seed, New England... 2.80 per 101 lbs. Quercus rubra, Red Oak, Northern Seed, New England..... 1.50 per 10 lbs.
$\$ 95.00$ per $100 \mathrm{lbs}$. 80.00 per 100 lbs. 22.00 per $100 \mathrm{lbs}$. 24.00 per $100 \mathrm{lbs}$. 10.00 per $100 \mathrm{lbs}$.

All our Oak Seed is hand selected.

Robinia pseudoacacia, Common Locust

$.75 \mathrm{lb} . \quad 75.00$ per $100 \mathrm{lbs}$.

\section{PLANTS FOR SHELTER AND FORAGE FOR ALL KINDS OF GAME}

Crataegus, American Hawthorns, dry berries of a number of species.. $\$ .25 \mathrm{lb} . \$ 20.00$ per $100 \mathrm{lbs}$. Morus alba tatarica, Russian Mulberry, a valuable forage plant for birds................................. $2.25 \mathrm{lb}$.

210.00 per 100 lbs.

Sorbus aucuparia, Mountain Ash, an important food tree for birds and wild fowl, in the fall and winter................ $.85 \mathbf{~ l b . ~}$

80.00 per $100 \mathrm{lbs}$. Siberian and Chinese Crab Apple, in a number of forms, fresh fruits. $.25 \mathrm{lb}$. Scotch Broom, Cytisus Scoparius..................... 1.10 1b. 20.00 per $100 \mathrm{lbs}$. 100.00 per $100 \mathrm{lbs}$.

Perennial Lupin, Lupinus perennis................. .85 lb. 80.00 per $100 \mathrm{lbs}$.

Our general list for tree and shrub seeds, as well as special list for Azalea and Rhododendron seeds are to be had for the asking. 
